\title{
Outcomes of surgical aortic valve replacement for severe aortic stenosis: Incorporation of left ventricular systolic function and stroke volume index
}

\author{
Roosha Parikh, MD, Andrew L. Goodman, MD, Tyler Barr, Joseph F. Sabik, MD, \\ Lars G. Svensson, MD, PhD, Luis Leonardo Rodriguez, MD, Bruce W. Lytle, MD, \\ Richard A. Grimm, MD, Brian P. Griffin, MD, and Milind Y. Desai, MD
}

\begin{abstract}
Objectives: We sought to assess predictors of mortality in consecutive patients with severe aortic stenosis undergoing aortic valve replacement and to determine whether there are differences in mortality, separated on the basis of different aortic stenosis subtypes and left ventricular stroke volume index.

Methods: We studied 875 patients (aged $69 \pm 12$ years, 67\% were men) with severe aortic stenosis (aortic valve area $\leq 1 \mathrm{~cm}^{2}$ ) who underwent aortic valve replacement between January 2007 and December 2008 (excluding other severe valve disease, balloon aortic valvuloplasty, and transcatheter aortic valve replacement). Clinical and echocardiographic data were recorded. Left ventricular stroke volume index was measured as left ventricular outflow tract velocity time integral $\times$ left ventricular outflow tract area/body surface area. Patients were classified into the following subtypes: (1) standard severe $(\mathrm{n}=536$, left ventricular ejection fraction $\geq 50 \%$ and mean gradient $\geq 40 \mathrm{~mm}$ $\mathrm{Hg})$; (2) paradoxic severe $(\mathrm{n}=152$, left ventricular ejection fraction $\geq 50 \%$, mean gradient $<40 \mathrm{~mm} \mathrm{Hg}$ and left ventricular stroke volume index $\left.<35 \mathrm{~mL} / \mathrm{m}^{2}\right)$; and (3) low left ventricular ejection fraction severe $(\mathrm{n}=187$, ejection fraction $<50 \%$ ). Society of Thoracic Surgeons score and all-cause mortality were recorded.
\end{abstract}

Results: At $4.8 \pm 2$ years, 153 patients (18\%) died (30-day mortality $1.8 \%)$. On multivariable Cox analysis, age (hazard ratio [HR], 1.49), New York Heart Association class (HR, 1.52), prior cardiac surgery (HR, 1.41), aortic stenosis subtypes (standard severe reference HR, 1; paradoxic severe HR, 1.48; and low left ventricular ejection fraction severe HR, 2.03), and reduced glomerular filtration rate $(\mathrm{HR}, 1.17)$ were associated with higher long-term mortality $(P<.05)$.

Conclusions: In patients with severe aortic stenosis undergoing aortic valve replacement, patients with standard severe aortic stenosis had better long-term survival than those with paradoxic severe or low left ventricular ejection fraction severe aortic stenosis. (J Thorac Cardiovasc Surg 2015;149:1558-66)

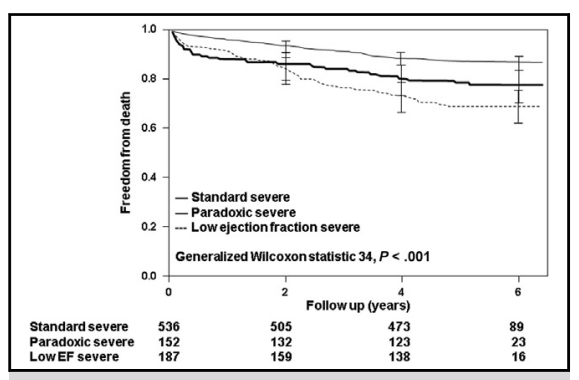

Kaplan-Meier survival curves of the study population are shown, divided on the basis of AS subtypes (standard severe, paradoxic severe, and low EF severe).

\section{Central Message}

In a contemporary study of patients with $\mathrm{AS}$ undergoing AVR, we demonstrate that despite low 30-day mortality, approximately $20 \%$ patients are dead at the 5-year follow-up. Patients with paradoxic severe and low LVEF severe AS had significantly worse outcomes than those with standard severe AS.

\section{Perspective}

In a contemporary study of patients with AS undergoing AVR, despite a low 30-day mortality, approximately 1 in 5 patients are dead at the 5 year follow-up. Along with expected predictors, such as increasing age, low glomerular filtration rate (GFR), and history of cardiac surgery, different AS subtypes and LV flow impairment were independently associated with mortality. Patients with paradoxic severe and low LVEF severe AS had significantly worse outcomes than those with standard severe AS. In patients with severe AS, a thorough clinical and imaging assessment needs to be performed to identify and characterize patients early, before the occurrence of advanced symptoms or LV flow impairment.

See Editorial page 1481.
From the Heart and Vascular Institute, Cleveland Clinic, Cleveland, Ohio. Drs Parikh and Goodman contributed equally to the work.

Received for publication Jan 5, 2015; revisions received Feb 25, 2015; accepted for publication March 7, 2015; available ahead of print April 11, 2015.

\footnotetext{
Address for reprints: Milind Y. Desai, MD, Department of Cardiovascular Medicine, Heart and Vascular Institute, Cleveland Clinic, 9500 Euclid Ave, Desk J1-5, Cleveland, OH 44195 (E-mail: desaim2@ccf.org). $0022-5223 / \$ 36.00$ Copyright (C) 2015 by The American Association for Thoracic Surgery http://dx.doi.org/10.1016/j.jtcvs.2015.03.008
} 


\author{
Abbreviations and Acronyms \\ AS = aortic stenosis \\ AVA $=$ aortic valve area \\ AVR = aortic valve replacement \\ $\mathrm{CABG}=$ coronary artery bypass grafting \\ $\mathrm{EF} \quad=$ ejection fraction \\ EOA $=$ effective orifice area \\ GFR $=$ glomerular filtration rate \\ $\mathrm{LV}=$ left ventricular \\ LVOT $=$ left ventricular outflow tract \\ LV-SVI = left ventricular stroke volume index \\ NYHA $=$ New York Heart Association \\ STS = Society of Thoracic Surgeons
}

Supplemental material is available online.

Over the years, numerous studies have demonstrated that aortic valve replacement (AVR) significantly improves survival in patients with severe aortic stenosis (AS). ${ }^{1-12}$ As a result, the current American College of Cardiology/ American Heart Association and European Society of Cardiology guidelines designate a class I indication for AVR in patients with severe AS who present with symptoms or who demonstrate signs of cardiac dysfunction, defined as resting left ventricular ejection fraction (LVEF) of $50 \%$ or less. 13,14

However, AS is a complex hemodynamic process, and determination of its true severity is frequently challenging. Although severe AS has been traditionally defined on the basis of echocardiographic aortic valve area (AVA) $1 \mathrm{~cm}^{2}$ or less and a mean $\mathrm{AV}$ gradient of $40 \mathrm{~mm} \mathrm{Hg}$ or more, ${ }^{15}$ it is increasingly recognized that not all patients with severe AS have severely elevated transvalvular gradients. Indeed, recent work has shown that the traditional definition of severe AS can fail to classify up to $15 \%$ to $30 \%$ of patients with reduced transvalvular flow gradients resulting from decreased LVEF or in those with normal LVEF because of diminished forward stroke volume related to myocardial remodeling, reduced left ventricular (LV) cavity size, or impaired LV compliance. ${ }^{16-18}$

Therefore, cardiologists increasingly are recognizing various subtypes of AS based not only on transvalvular gradient, valve area, and ejection fraction (EF) but also on LV stroke volume index (LV-SVI). Accurate characterization of patients as having standard severe, paradoxic severe, and low LVEF low gradient severe AS, based on AVA, LVEF, transvalvular gradients, and LV-SVI, is likely important, because these groups of patients may have varying outcomes as demonstrated in prior moderately sized studies. ${ }^{2,3,17,19-24}$ Many prior studies have confined their attention to patients undergoing isolated AVR. Although this may make analysis of outcomes simpler, it fails to recognize that many of the patients currently undergoing operation for AS have concomitant coronary disease or disease of the aorta that significantly affects their operative and subsequent course and is an inherent part of the disease spectrum of AS in the current era. In the current study, we sought to (1) assess predictors of mortality in a consecutive contemporary group of patients with severe AS undergoing AVR and (2) determine whether there are differences in mortality, separated on the basis of different AS subtypes and preserved versus impaired LV-SVI.

\section{MATERIALS AND METHODS \\ Study Population}

This was an observational cohort study of 875 consecutive patients with severe AS (AVA $\leq 1 \mathrm{~cm}^{2}$ ) who had an echocardiogram at our tertiary care center between January 2007 and December 2008 followed by AVR at Cleveland Clinic. We excluded the following patients: those with other valvular disease (ie, mitral/tricuspid valve disease or prosthetic AS) and those who underwent transcatheter AVR or balloon aortic valvuloplasty (because, at the time, these therapies were performed in individuals who were deemed high risk or inoperable).

\section{Clinical Data}

Data were assembled by analysis of electronic medical records after appropriate approval by the institutional review board was obtained. Demographics and clinical data were extracted from the electronic health record at the time of the initial visit. The presence of angina, syncope, and New York Heart Association (NYHA) class was extracted from the electronic medical record to assess the symptomatic status of each patient. The decision to operate was made after a careful evaluation by the cardiologist and cardiothoracic surgeon at the time of initial presentation. No patient went directly for AVR without being evaluated by a cardiologist at our center. In patients who were perceived to be asymptomatic at the initial evaluation, the decision to operate was made on the basis of the following potential high-risk factors: LV systolic dysfunction, abnormal blood pressure response, excessive LV hypertrophy, and AVA less than $0.6 \mathrm{~cm}^{2}$. On the basis of the available preoperative data, Society of Thoracic Surgeons (STS) score was calculated.

\section{Preoperative Echocardiography}

All patients underwent a comprehensive echocardiogram with commercially available instruments (Philips Medical Systems, NA, Bothell, Wash; General Electric Medical Systems, Milwaukee, Wis; and Siemens Medical Solutions USA, Inc, Malvern, Pa) as part of a standard clinical diagnostic evaluation. Measurements and recordings were obtained according to current recommendations. ${ }^{15,25} \mathrm{LV}$ dimensions (end systolic and end diastolic), LV mass, and left atrial dimensions were measured from 2-dimensional images and indexed to body surface area. LVEF was calculated using the Simpson's biplane method. Diastolic function was graded using the current grading recommendations for this assessment. ${ }^{26}$ We used a semiquantitative 5-point scale (with grades of none, mild, moderate, moderately severe, and severe) to stratify aortic regurgitation, mitral regurgitation, and tricuspid regurgitation assessed by color 2-dimensional Doppler echocardiography clips obtained in multiple standard views. Right ventricular systolic pressure was estimated in a standard fashion. ${ }^{27}$ 
For quantification of AS severity, LV outflow tract diameter was measured in a standard fashion on parasternal long-axis views. ${ }^{15}$ The AVA was calculated using the continuity equation. Dimensionless index was calculated as left ventricular outflow tract

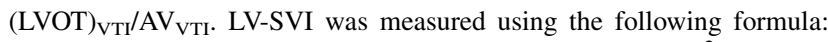
LVOT $_{\text {VTI }} \times$ LVOT $_{\text {area }} /$ body surface area. A cutoff of $35 \mathrm{~mL} / \mathrm{m}^{2}$ or greater was considered as preserved LV-SVI. ${ }^{15-18}$ On the basis of these calculations, severe AS was defined as AVA $1 \mathrm{~cm}^{2}$ or less and dimensionless index less than 0.25 . The patients in the study population were further classified as having (1) standard severe AS (AVA $\leq 1 \mathrm{~cm}^{2}$, LVEF $\geq 50 \%$, dimensionless index $<0.25$, and mean gradient $\geq 40 \mathrm{~mm}$ $\mathrm{Hg}$ ); (2) paradoxical severe AS (AVA $\leq 1 \mathrm{~cm}^{2}$, LVEF $\geq 50 \%$, mean gradient $<40 \mathrm{~mm} \mathrm{Hg}$, dimensionless index $<0.25$, and LV-SVI $<35 \mathrm{~mL} / \mathrm{m}^{2}$ ); and (3) low EF severe AS (AVA $\leq 1 \mathrm{~cm}^{2}$, mean gradient $<40 \mathrm{~mm} \mathrm{Hg}$, dimensionless index $<0.25$, and $\mathrm{EF}<50 \%$ ). ${ }^{16,19}$

\section{Surgery}

The details of various surgical therapies for the management of AS were recorded and categorized as follows: (1) AVR; (2) AVR and coronary artery bypass grafting (CABG); (3) AVR and concomitant other valve repair/replacement (which is standard practice if the other valvular lesion is moderate) \pm CABG; and (4) AVR and ascending aorta repair or replacement \pm CABG. In patients with moderate disease of other valves (mitral or tricuspid), in addition to severe AS, additional valve surgery (most commonly mitral or tricuspid valve repair \pm CABG) was also performed at the time of operation. The final decision regarding the specific operative technique was made by the attending cardiothoracic surgeon.

\section{Postoperative Echocardiography}

We evaluated postoperative follow-up echocardiograms for LVEF and $\mathrm{AV}$ gradients. In addition, we recorded different types and sizes of $\mathrm{AV}$ prostheses, including bioprostheses, mechanical prostheses, and homografts. Subsequently, to determine the presence of patient-prosthesis mismatch, we recorded the effective orifice areas (EOAs) of the prosthetic $\mathrm{AV}$ (stroke volume/prosthetic valve $\mathrm{VTI}_{\mathrm{VT}}$ ) in each patient and indexed it to body surface area. ${ }^{28}$ Normal indexed EOA values are greater than 0.85 $\mathrm{cm}^{2} / \mathrm{m}^{2}$. Patient-prosthesis mismatch was categorized as severe if indexed EOA was less than $0.65 \mathrm{~cm}^{2} / \mathrm{m}^{2}$ and moderate if indexed EOA was between 0.65 and $0.85 \mathrm{~cm}^{2} / \mathrm{m}^{2}{ }^{28}$

\section{Outcomes Assessment}

All-cause mortality was considered to be the primary outcome. Death notification was confirmed by observation of death certificate or verified with a family member. The duration of follow-up ranged between time of AVR and June 2013. In addition, we also recorded postoperative stroke, defined as a neurologic impairment lasting more than 24 hours because of cerebral ischemia or hemorrhage confirmed by radiographic studies. Transient ischemic attack was similarly defined as neurologic impairment lasting less than 24 hours.

\section{Statistical Analysis}

Continuous variables are expressed as mean \pm standard deviation or median and compared using Student $t$ test or analysis of variance (for normally distributed variables) or Mann-Whitney test (for non-normally distributed variables). Categoric data are expressed as percentage and compared using the chi-square test. To assess outcomes, Cox proportional hazards analysis was used. Initially, univariable analysis was performed for individual potential predictors of mortality. Subsequently, forward stepwise multivariable survival analysis was performed incorporating only selected and relevant variables, known to be associated with outcomes in patients with AS. For the stepwise analysis, a $P$ value threshold less than .1 was used as a model entry cutoff. Hazard ratios with $95 \%$ confidence intervals were calculated. The proportional hazards assumption was examined using inspection of Schoenfeld residuals plotted against time. Also, to test the linearity of the relationship between STS score and survival in the Cox proportional hazards model, we performed polynomial transformations of STS score. Survival, as a function over time, was analyzed using the Kaplan-Meier method, and event curves were analyzed using a log-rank test in which proportional hazards were not violated and a generalized Wilcoxon (Breslow's) test in which the survival curves clearly crossed and violated the proportional hazards. Statistical analysis was performed using SPSS version 11.5 (SPSS Inc, Chicago, Ill), Stata version 10.0 (StataCorp, LP, College Station, Tex), and R 3.0.3 (R foundation for Statistical Computing, Vienna, Austria).

\section{RESULTS}

The baseline and clinical data in the study population as a whole and divided into various AS subgroups are shown in Table 1. This was an older population with an expected distribution of cardiovascular risk factors. However, because this was a contemporary study population, the majority of the patients were taking optimal cardioprotective medications, with expected differences between subgroups that had preserved versus low LVEF. No patient had active endocarditis at the time of AVR. The echocardiographic data in the study population as a whole and divided into various AS subgroups are shown in Table 2. As expected, the LV size parameters were abnormal in the subgroup with low LVEF severe AS and normal in the other 2 subgroups. AV gradients were the highest in the standard severe AS subgroup, whereas patients with low LVEF severe AS had the highest proportion of moderate mitral and tricuspid regurgitation.

The distribution of cardiac surgeries in the study population as a whole and divided into various AS subgroups is shown in Table 3. The highest proportion of isolated AVR was performed in the standard severe AS subgroup, and the highest proportion of concomitant CABG was performed in the low EF severe AS subgroup. Distribution of different valve types was as follows: bioprosthesis $(92 \%)$, mechanical prosthesis $(6 \%)$, and homograft $(1 \%)$, with no differences between subgroups.

\section{Outcomes and Survival Assessment}

During a mean follow up of $4.8 \pm 2$ years, 153 patients $(18 \%)$ died. At 30 days postoperatively, 16 patients $(1.8 \%)$ died, of whom 9 were undergoing redo cardiac surgery (4 patients $[1 \%]$ in standard severe, 6 patients [4\%] in paradoxic severe, and 6 patients [3\%] in low LVEF severe AS subgroups). Only 2 patients undergoing isolated AVR died at 30 days, whereas the remainder of deaths occurred in those undergoing a combination procedure (AVR in addition to other surgery). In addition, 44 patients $(5 \%)$ died at 1 year and 66 patients $(8 \%)$ died at 2 years, with no differences in mortality between different surgeries or AS subtypes. Stroke within 30 days postoperatively was observed in 12 patients $(1.3 \%)$. There 
TABLE 1. Baseline characteristics of the study population

\begin{tabular}{|c|c|c|c|c|c|}
\hline & $\begin{array}{c}\text { Total population } \\
(\mathbf{n}=\mathbf{8 7 5})\end{array}$ & $\begin{array}{c}\text { Normal EF, } \\
\text { high gradient }(n=536)\end{array}$ & $\begin{array}{c}\text { Normal EF, low gradient } \\
\text { paradoxic }(n=152)\end{array}$ & $\begin{array}{c}\text { Low EF, } \\
\text { low gradient }(n=187)\end{array}$ & $P$ value \\
\hline Age (y) & $69 \pm 12$ & $69 \pm 12$ & $69 \pm 12$ & $72 \pm 11$ & .004 \\
\hline Male gender & $584(67 \%)$ & $337(63 \%)$ & $96(63 \%)$ & $151(81 \%)$ & $<.001$ \\
\hline $\operatorname{BSA}\left(\mathrm{m}^{2}\right)$ & $2.0 \pm 0.3$ & $2.0 \pm 0.3$ & $2.0 \pm 0.3$ & $2.1 \pm 0.3$ & .5 \\
\hline Angina & $296(34 \%)$ & $187(35 \%)$ & $50(33 \%)$ & $59(32) \%$ & .2 \\
\hline Syncope & $61(17 \%)$ & $36(7 \%)$ & $7(5 \%)$ & $18(10 \%)$ & .13 \\
\hline NYHA class & & & & & $<.001$ \\
\hline I & $164(19 \%)$ & $111(21 \%)$ & $31(20 \%)$ & $22(12 \%)$ & \\
\hline II & $402(46 \%)$ & $266(50 \%)$ & $60(40 \%)$ & $76(41 \%)$ & \\
\hline III & $264(30 \%)$ & $143(27 \%)$ & $54(36 \%)$ & $67(36 \%)$ & \\
\hline IV & $45(5 \%)$ & $16(3 \%)$ & $7(4 \%)$ & $22(12 \%)$ & \\
\hline Hypertension & $664(76 \%)$ & $423(79 \%)$ & $124(82 \%)$ & $117(63 \%)$ & $<.001$ \\
\hline Hyperlipidemia & $737(83 \%)$ & $448(84 \%)$ & $131(86 \%)$ & $158(85 \%)$ & .7 \\
\hline Diabetes mellitus & $210(24 \%)$ & $115(22 \%)$ & $39(26 \%)$ & $56(30 \%)$ & .06 \\
\hline Prior stroke & $66(8 \%)$ & $41(8 \%)$ & $15(10 \%)$ & $10(5 \%)$ & .29 \\
\hline Smoking history & $444(51 \%)$ & $271(51 \%)$ & $86(54 \%)$ & $87(47 \%)$ & .3 \\
\hline Obstructive CAD & $512(59 \%)$ & $283(53 \%)$ & $104(68 \%)$ & $125(67 \%)$ & $<.001$ \\
\hline Atrial fibrillation & $182(21 \%)$ & $102(19 \%)$ & $30(20 \%)$ & $50(27 \%)$ & .07 \\
\hline Dialysis & $22(3 \%)$ & $8(2 \%)$ & $5(3 \%)$ & $9(5 \%)$ & .04 \\
\hline Prior open surgery & $179(20 \%)$ & $85(16 \%)$ & $28(18 \%)$ & $66(35 \%)$ & $<.001$ \\
\hline ICD & $51(6 \%)$ & $14(3 \%)$ & $4(3 \%)$ & $33(18 \%)$ & $<.001$ \\
\hline Pacemaker & $431(49 \%)$ & $257(48 \%)$ & $73(48 \%)$ & $101(54 \%)$ & .3 \\
\hline STS score $(\%)$ & $5.4 \pm 6$ & $4.6 \pm 6$ & $5.1 \pm 6$ & $8.0 \pm 8$ & $<.001$ \\
\hline Beta-blockers & $810(93 \%)$ & $494(92 \%)$ & $139(91 \%)$ & $177(95 \%)$ & .45 \\
\hline ACE inhibitors & $421(48 \%)$ & $215(40 \%)$ & $79(52 \%)$ & $127(68 \%)$ & $<.001$ \\
\hline Aspirin & $819(94 \%)$ & $501(94 \%)$ & $141(93 \%)$ & $177(95 \%)$ & .76 \\
\hline Statins & $700(80 \%)$ & $426(80 \%)$ & $125(82 \%)$ & $149(80 \%)$ & .7 \\
\hline Diuretics & $825(94 \%)$ & $505(94 \%)$ & $141(93 \%)$ & $179(96 \%)$ & .002 \\
\hline Aldosterone receptor blocker & $93(11 \%)$ & $39(7 \%)$ & $10(7 \%)$ & $44(24 \%)$ & $<.001$ \\
\hline Hemoglobin (mg/dL) & $13 \pm 2$ & $14 \pm 2$ & $13 \pm 2$ & $13 \pm 2$ & .1 \\
\hline $\operatorname{GFR}\left(\mathrm{mL} / \mathrm{min} / 1.73 \mathrm{~m}^{2}\right)$ & $69 \pm 22$ & $70 \pm 20$ & $68 \pm 23$ & $64 \pm 24$ & .001 \\
\hline $\mathrm{LDL}(\mathrm{mg} / \mathrm{dL})$ & $98 \pm 37$ & $100 \pm 34$ & $100 \pm 46$ & $94 \pm 40$ & .1 \\
\hline HDL (mg/dL) & $50 \pm 16$ & $51 \pm 16$ & $52 \pm 15$ & $45 \pm 15$ & .001 \\
\hline
\end{tabular}

$P$ values reflect comparison between AS subtypes. $E F$, Ejection fraction; BSA, body surface area; NYHA, New York Heart Association; $C A D$, coronary artery disease; $I C D$, internal cardioverter defibrillator; STS, Society of Thoracic Surgery; $A C E$; angiotensin-converting enzyme; GFR, glomerular filtration rate; $H D L$, high-density lipoprotein; $L D L$, low-density lipoprotein.

were an additional 14 strokes $(1.6 \%)$ and 6 transient ischemic attacks $(0.7 \%)$ during follow-up. Transient atrial fibrillation (within 30 days postoperatively) was observed in 123 patients $(14 \%)$. Postoperative pacemaker and implantable cardioverter defibrillators were implanted in 35 patients $(4 \%)$ and 9 patients $(1 \%)$, respectively.

Subsequently, univariable and forward stepwise multivariable Cox proportional hazard analysis was performed for the primary end point of long-term all-cause mortality. Neither quadratic nor cubic transformations of STS score were significant predictors of long-term mortality when forced into the Cox model that already included nontransformed STS score. Initially, individual predictors, potentially associated with mortality in patients with AS, were tested in a univariable fashion. The results are shown in Table E1. Subsequently, only selected relevant predictors were entered into a forward multivariable stepwise model, the results of which are shown in Table 4. Atrial fibrillation, coronary artery disease, LV mass index, and patient-prosthesis mismatch were associated with long-term all-cause mortality on univariable analysis; however, they did not remain independently significant on multivariable survival analysis. Also, the type of cardiac surgery performed was not associated with long-term outcomes. When STS score was entered into the multivariable model (instead of its individual predictors), the results were similar (Table 4, model B). The proportion of long-term all-cause deaths in the various AS subtypes were as follows: 65 of 536 $(12 \%)$ in the standard severe AS subgroup, 32 of 152 $(21 \%)$ in the paradoxic severe AS subgroup, and 56 of $187(30 \%)$ in the low LVEF severe AS subgroup. Kaplan-Meier survival curves, demonstrating the mortality in the study population, separated on the basis of different 
TABLE 2. Baseline and follow-up echocardiographic characteristics of the study population

\begin{tabular}{|c|c|c|c|c|c|}
\hline & $\begin{array}{l}\text { Total population } \\
\qquad(\mathrm{n}=\mathbf{8 7 5})\end{array}$ & $\begin{array}{c}\text { Normal EF, high } \\
\text { gradient }(n=536)\end{array}$ & $\begin{array}{l}\text { Normal EF, low gradient } \\
\text { paradoxic }(\mathbf{n}=152)\end{array}$ & $\begin{array}{c}\text { Low EF, low } \\
\text { gradient }(n=187)\end{array}$ & $P$ value \\
\hline \multicolumn{6}{|l|}{ Preoperative echocardiography } \\
\hline $\operatorname{LVEF}(\%)$ & $59 \pm 5$ & $59 \pm 5$ & $57 \pm 4$ & $34 \pm 10$ & $<.001$ \\
\hline Indexed LVEDD $\left(\mathrm{cm} / \mathrm{m}^{2}\right)$ & $2.4 \pm 0.4$ & $2.3 \pm 0.4$ & $2.3 \pm 0.4$ & $2.7 \pm 0.4$ & $<.001$ \\
\hline Indexed LVESD $\left(\mathrm{cm} / \mathrm{m}^{2}\right)$ & $1.6 \pm 0.5$ & $1.4 \pm 0.3$ & $1.4 \pm 0.3$ & $2.0 \pm 0.6$ & $<.001$ \\
\hline Indexed LA dimension $\left(\mathrm{cm} / \mathrm{m}^{2}\right)$ & $2.1 \pm 0.4$ & $2.1 \pm 0.4$ & $2.1 \pm 0.4$ & $2.3 \pm 0.4$ & $<.001$ \\
\hline Indexed LV mass $\left(\mathrm{g} / \mathrm{m}^{2}\right)$ & $126 \pm 43$ & $129 \pm 42$ & $128 \pm 41$ & $149 \pm 46$ & $<.001$ \\
\hline Diastolic dysfunction & & & & & $<.001$ \\
\hline Abnormal relaxation & $735(84 \%)$ & $461(86 \%)$ & $134(88 \%)$ & $140(74 \%)$ & \\
\hline Pseudo-normal & $123(14 \%)$ & $70(13 \%)$ & $18(12 \%)$ & $35(20 \%)$ & \\
\hline Restrictive filling & $17(2 \%)$ & $5(1 \%)$ & 0 & $2(6 \%)$ & \\
\hline LVOT diameter $(\mathrm{cm})$ & $2.0 \pm 0.2$ & $2.0 \pm 0.2$ & $2.0 \pm 0.2$ & $2.0 \pm 0.2$ & .3 \\
\hline \multicolumn{6}{|l|}{$\mathrm{AV}$ gradient } \\
\hline Peak $(\mathrm{mm} \mathrm{Hg})$ & $81 \pm 27$ & $94 \pm 22$ & $56 \pm 13$ & $64 \pm 24$ & $<.001$ \\
\hline Mean $(\mathrm{mm} \mathrm{Hg})$ & $47 \pm 16$ & $55 \pm 13$ & $31 \pm 7$ & $37 \pm 15$ & $<.001$ \\
\hline Calculated $\mathrm{AV}$ area (continuity equation) & $0.70 \pm 0.2$ & $0.67 \pm 0.2$ & $0.76 \pm 0.1$ & $0.75 \pm 0.2$ & $<.001$ \\
\hline LV-SVI $\left(\mathrm{mL} / \mathrm{m}^{2}\right)$ & $37 \pm 10$ & $40 \pm 10$ & $29 \pm 4$ & $32 \pm 9$ & $<.001$ \\
\hline $\begin{array}{l}\text { Aortic dimension at mid-ascending } \\
\text { segment }(\mathrm{cm})\end{array}$ & $3.4 \pm 0.6$ & $3.4 \pm 0.6$ & $3.3 \pm 0.6$ & $3.4 \pm 0.6$ & .3 \\
\hline Aortic regurgitation & & & & & .5 \\
\hline None & $162(19 \%)$ & $100(19 \%)$ & $30(20 \%)$ & $32(17 \%)$ & \\
\hline Mild & $495(56 \%)$ & $307(57 \%)$ & $88(55 \%)$ & $100(53 \%)$ & \\
\hline Moderate & $155(18 \%)$ & $94(18 \%)$ & $22(15 \%)$ & $39(21 \%)$ & \\
\hline Moderate-severe & $49(6 \%)$ & $26(5 \%)$ & $10(7 \%)$ & $13(7 \%)$ & \\
\hline Severe & $14(2 \%)$ & $9(2 \%)$ & $2(2 \%)$ & $3(2 \%)$ & \\
\hline Mitral regurgitation & & & & & $<.001$ \\
\hline None & $101(12 \%)$ & $64(12 \%)$ & $23(15 \%)$ & $14(7 \%)$ & \\
\hline Mild & $625(72 \%)$ & $413(77 \%)$ & $108(71 \%)$ & $104(59 \%)$ & \\
\hline Moderate & $149(17 \%)$ & $59(12 \%)$ & $21(14 \%)$ & $69(35 \%)$ & \\
\hline Tricuspid regurgitation & & & & & $<.001$ \\
\hline None & $105(12 \%)$ & $64(12 \%)$ & $20(13 \%)$ & $21(10 \%)$ & \\
\hline Mild & $691(79 \%)$ & $434(81 \%)$ & $117(77 \%)$ & $140(71 \%)$ & \\
\hline Moderate & $79(9 \%)$ & $38(7 \%)$ & $15(10 \%)$ & $26(14 \%)$ & \\
\hline RVSP (mm Hg) & $36 \pm 11$ & $35 \pm 11$ & $34 \pm 11$ & $41 \pm 16$ & $<.001$ \\
\hline \multicolumn{6}{|l|}{ Postoperative echocardiography } \\
\hline $\operatorname{LVEF}(\%)$ & $51 \pm 11$ & $53 \pm 7$ & $54 \pm 7$ & $38 \pm 13$ & $<.001$ \\
\hline \multicolumn{6}{|l|}{$\mathrm{AV}$ gradient } \\
\hline Peak $(\mathrm{mm} \mathrm{Hg})$ & $25 \pm 11$ & $27 \pm 12$ & $24 \pm 9$ & $23 \pm 10$ & $<.001$ \\
\hline Mean $(\mathrm{mm} \mathrm{Hg})$ & $14 \pm 6$ & $15 \pm 7$ & $13 \pm 5$ & $13 \pm 6$ & $<.001$ \\
\hline Indexed EOA of prosthetic valve $\left(\mathrm{cm}^{2} / \mathrm{m}^{2}\right)$ & $0.88 \pm 0.14$ & $0.88 \pm 0.11$ & $0.86 \pm 0.12$ & $0.92 \pm 0.14$ & .005 \\
\hline Patient prosthesis mismatch & & & & & .02 \\
\hline None & $59 \%$ & $304(58 \%)$ & $77(52 \%)$ & $124(68 \%)$ & \\
\hline Moderate & $336(40 \%)$ & $213(41 \%)$ & $66(45 \%)$ & $57(31 \%)$ & \\
\hline Severe & $10(1 \%)$ & $5(1 \%)$ & $4(3 \%)$ & $1(0.5 \%)$ & \\
\hline
\end{tabular}

$P$ values reflect comparison between AS subtypes. $E F$, Ejection fraction; $L V E F$, left ventricular ejection fraction; $L V E D D$, left ventricular end-diastolic dimension; $L V E S D$, left ventricular end-systolic dimension; $L A$, left atrium; $L V$, left ventricular; $L V O T$, left ventricular outflow tract; $A V$, aortic valve; $L V$-SVI, left ventricular stroke volume index; RVSP, right ventricular systolic pressure; $E O A$, effective orifice area.

AS subtypes are shown in Figure 1 (generalized Wilcoxon statistic 34, $P<.001$ ). Likewise, the proportion of all-cause deaths in the population, separated on the basis of preserved $\left(\geq 35 \mathrm{~mL} / \mathrm{m}^{2}\right)$ versus impaired $\left(<35 \mathrm{~mL} / \mathrm{m}^{2}\right)$ LV-SVI were as follows: 62 of $439(14 \%)$ in those with preserved LV-SVI versus 91 of $436(21 \%)$ in those with impaired LV-SVI. Kaplan-Meier survival curves, demonstrating the mortality in the study population, separated on the basis of preserved versus impaired LV-SVI, are shown in Figure 2 (log-rank statistic 7, $P=.007)$.

\section{DISCUSSION}

In the current contemporary study of patients with severe AS undergoing AVR (isolated or in combination with other surgical procedures), we divided the population into various 
TABLE 3. Distribution of cardiac surgeries in the total study population and various aortic stenosis subgroups

\begin{tabular}{|c|c|c|c|c|c|}
\hline & $\begin{array}{l}\text { Total population } \\
\quad(\mathbf{n}=875)\end{array}$ & $\begin{array}{c}\text { Normal EF, } \\
\text { high gradient }(n=536)\end{array}$ & $\begin{array}{l}\text { Normal EF, low gradient } \\
\text { paradoxic }(\mathbf{n}=152)\end{array}$ & $\begin{array}{c}\text { Low EF, low gradient } \\
(\mathbf{n}=187)\end{array}$ & $P$ value \\
\hline Isolated AVR & $390(45 \%)$ & $279(52 \%)$ & $55(36 \%)$ & $56(30 \%)$ & $<.001$ \\
\hline $\mathrm{AVR}+\mathrm{CABG}$ & $312(36 \%)$ & $163(30 \%)$ & $67(44 \%)$ & $82(44 \%)$ & \\
\hline $\begin{array}{l}\text { AVR }+ \text { other valve repair } \\
\text { or replacement } \pm \text { CABG }\end{array}$ & $95(11 \%)$ & $37(7 \%)$ & $19(13 \%)$ & $39(21 \%)$ & \\
\hline $\begin{array}{l}\mathrm{AVR}+\text { ascending aorta repair } \\
\text { or replacement } \pm \mathrm{CABG}\end{array}$ & $78(9 \%)$ & $57(11 \%)$ & $11(7 \%)$ & $10(5 \%)$ & \\
\hline
\end{tabular}

subtypes (standard severe, paradoxic severe, and low EF severe AS) on the basis of AV gradients, LVEF, and LV-SVI, excluding those with low AV gradients and preserved LV-SVI. To the best of our knowledge, this is one of the largest studies evaluating predictors of outcomes of such patients, incorporating different AS subtypes and LV-SVI. As expected, this was an older population with an expected distribution of cardiovascular risk factors among different AS subtypes. Because this was a contemporary study population, the majority of the patients were taking optimal cardioprotective medications. Only $45 \%$ of patients underwent isolated AVR; the rest underwent a combination procedure, with coronary bypass

TABLE 4. Forward stepwise multivariable Cox proportional hazards analysis for the primary end point of long-term mortality

\begin{tabular}{lcc}
\hline \multicolumn{1}{c}{ Variable } & Hazard ratio & $\boldsymbol{P}$ value \\
\hline Model A & & \\
Age (10-y increase) & $1.49(1.27-1.75)$ & $<.001$ \\
NYHA class & $1.52(1.22-1.90)$ & $<.001$ \\
GFR (for every 10-unit decrease) & $1.17(1.08-1.27)$ & $<.001$ \\
Prior cardiac surgery & $1.41(1.05-1.96)$ & .03 \\
AS subcategories & & \\
$\quad$ Standard severe & 1 & \\
$\quad$ Paradoxic severe & $1.48(1.15-188)$ & $<.001$ \\
$\quad$ Low EF severe & $2.03(1.41-2.93)$ & $<.001$ \\
Chi-square for the model $=97, P<.0001$ & \\
Model B & & \\
STS score (for every 1\% increase) & $1.35(1.15-1.58)$ & $<.001$ \\
AS subcategories & & \\
$\quad$ Standard severe & 1 & $<.001$ \\
$\quad$ Paradoxic severe & $1.46(1.15-1.86)$ & \\
$\quad$ Low EF severe & $2.26(1.70-3.36)$ & $<.001$ \\
Chi-square for the model 53, $P<.001$ & & \\
\hline
\end{tabular}

In model $\mathrm{A}$, the following predictors were considered for analysis: age, gender, symptoms, coronary artery disease, atrial fibrillation, prior cardiac surgery, GFR, indexed LV mass, aortic regurgitation, right ventricular systolic pressure, type of cardiac surgery performed, time to cardiac surgery, type of aortic valve prosthesis implanted, and patient-prosthesis mismatch. Because of collinearity with AS subtypes, LV-SVI and LVEF were not included in the analysis. However, if they were substituted for AS subtypes, LV-SVI and LVEF were significant. Patient-prosthesis mismatch (and not indexed EOA) was included in the multivariable analysis. Results were similar if indexed EOA was substituted for patient-prosthesis mismatch. In model B, predictors that constituted part of STS score were not considered for analysis. Other predictors were similar to model A. NYHA, New York Heart Association; GFR, glomerular filtration rate; $A S$, aortic stenosis; $E F$, ejection fraction; STS, Society of Thoracic Surgeons. grafting being the most common concomitant surgery performed. Postoperative echocardiography revealed successful reduction in AV gradients and a low incidence of severe patient-prosthesis mismatch, with no significant differences between AS subtypes.

In the current study, despite a low 30-day postoperative mortality, approximately 1 in 5 patients have died during $4.8 \pm 2$ years of follow-up, suggesting that patients with severe AS represent a sick cohort. As expected, long-term mortality was independently associated with increasing age, worsening NYHA class, reduced renal function, and need for redo cardiac surgery (or STS score, which is a composite of multiple risk factors). In addition, we also demonstrate that patients with different AS subtypes have significant differences in mortality after AVR. Those with standard severe AS had significantly better outcomes compared with those with paradoxic or low LVEF severe AS. Incorporating AS subtypes or LV-SVI resulted in significant improvement in predicting long-term survival over and above STS score (or standard individual clinical predictors such as NYHA class and age). Although other potential variables, such as atrial fibrillation, coronary artery disease, indexed LV mass (a surrogate of LV hypertrophy), and patient-prosthesis mismatch, were significantly associated with mortality on univariable analysis, they did not remain independently predictive on

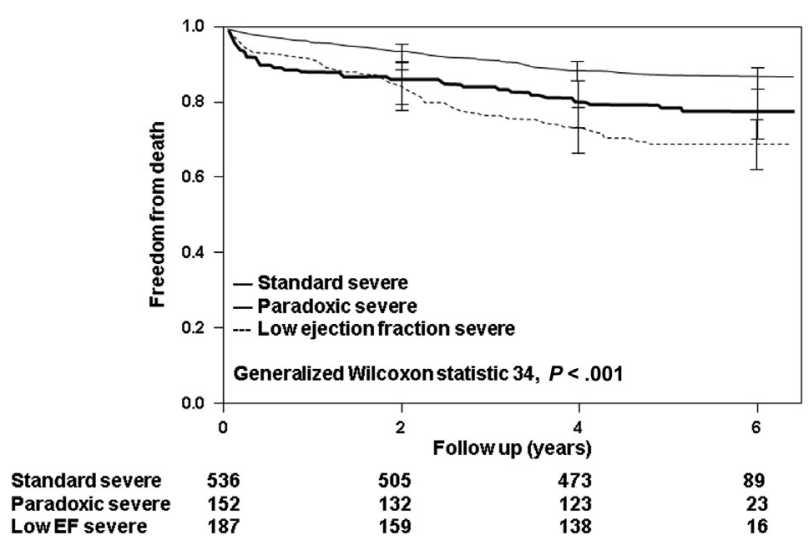

FIGURE 1. Kaplan-Meier survival curves of the study population, divided on the basis of AS subtypes. $E F$, Ejection fraction. 


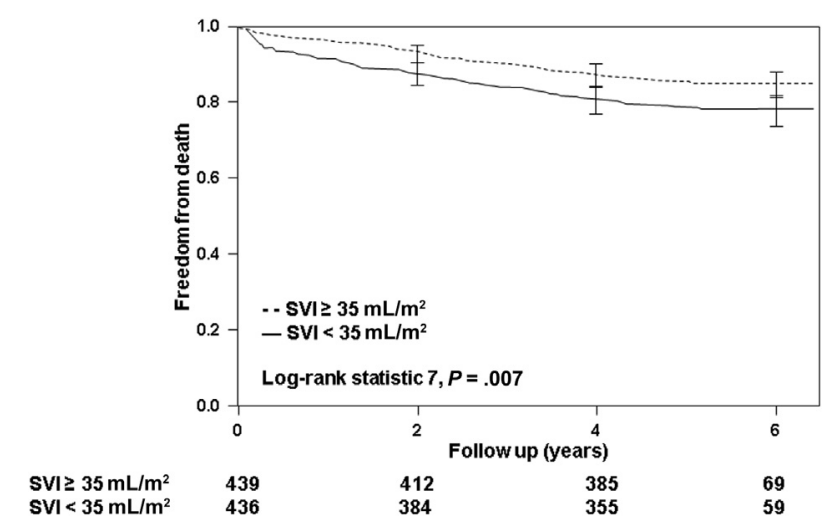

FIGURE 2. Kaplan-Meier survival curves of the study population, divided on the basis of preserved versus impaired LV-SVI. SVI, Stroke volume index.

multivariable analysis. The results were similar when documented noncardiac causes of mortality (eg, cancer, neurologic etiologies, advanced renal/liver diseases) were excluded from survival analysis. These data suggest that in patients with severe AS, a thorough clinical and imaging assessment needs to be performed to identify patients early, before occurrence of advanced symptoms or LV flow impairment. In such patients, early AVR could be associated with improved survival. However, this observation requires further prospective validation.

Similar to our study, multiple previous studies have reported that AVR results in improved survival in patients with severe AS, along with its potential predictors. ${ }^{1-12}$ However, unlike the current study, many of these studies are older, had relatively small sample sizes, or only reported outcomes of isolated AVR. Because we included only patients from 2007 and 2008, the current study took into account only modern surgical techniques, and the majority of the patients were receiving appropriate cardioprotective medications known to be associated with improved survival. Furthermore, unlike the other studies, the current study used Doppler echocardiography to distinguish between various AS subtypes and flow characteristics based on AVA, dimensionless index, AV gradients, LVEF, and LV-SVI. Indeed, previous studies have reported that assessment of transvalvular flow is an important determinant of outcome, and patients with a reduced gradient, despite preserved LVEF, had poor outcomes with medical therapy compared with AVR. ${ }^{16-19}$ However, unlike those previous reports, all patients in the current study underwent AVR, and we demonstrate that patients with paradoxic severe AS have a higher mortality when compared with those with standard severe AS. As expected, increasing age, history of cardiac surgery, and low GFR were associated with worse outcomes. However, unlike previous reports that have suggested that patientprosthesis mismatch and LV hypertrophy predicted increased mortality, ${ }^{8,29}$ in the current study, these variables did not remain independently predictive on multivariable analysis. This is most likely because of a low incidence of severe patient-prosthesis mismatch in the current study, likely due to a more modern population. Another possibility is that LV flow and systolic function are stronger predictors of outcomes than these variables.

\section{Clinical Implications}

In a contemporary study of patients with AS undergoing AVR, we demonstrate that despite low 30-day mortality, approximately 1 in 5 patients are dead at the 5-year follow-up. Along with the expected predictors such as increasing age, low GFR, and a history of cardiac surgery, different AS subtypes (and LV flow impairment) were also independently associated with mortality. Patients with paradoxic severe and low LVEF severe AS had significantly worse outcomes compared with those with standard severe AS. In patients with severe AS, a thorough clinical and imaging assessment needs to be performed to identify and characterize patients early, before occurrence of advanced symptoms or LV flow impairment. Early AVR (eg, before development of advanced symptoms) could be associated with improved outcomes in this high-risk population.

\section{Study Strengths and Limitations}

This is an observational study from a single tertiary care center, and thus is not free from selection bias. In addition, some of the higher-risk patients undergoing evaluation for surgical AVR could have been diverted to transcatheter AVR. We can only report associations, not causality. We only selected patients who were initially evaluated (clinically and by echocardiography) in 2007 and 2008. This inherently allowed us to focus on a contemporary population with a high proportion of cardioprotective medications, in whom surgery was performed over a narrow time frame using similar, modern surgical techniques. Unlike in previous reports, our study population was heterogeneous and did not just consist of patients who underwent isolated AVR. Although this may make analysis of outcomes simpler, many of the patients currently undergoing operation for AS have concomitant coronary disease or disease of the aorta that significantly affects their operative and subsequent course and is an inherent part of the disease spectrum of AS in the current era. Because echocardiographic measurements of LVOT diameter and $\mathrm{AV}$ gradients are operator-dependent, there is the likelihood that some patients with moderate AS were misclassified as having paradoxic severe AS. However, despite that possibility, the outcomes were significantly worse in the paradoxic AS subtype compared with the standard severe AS subtype. That is likely the reason why incorporating flow assessment (ie, LV-SVI) in the workup of the patient 
could help further refine the classification of these patients. Another limitation arises when continuous variables such as $\mathrm{AV}$ gradient and LV-SVI are dichotomized. However, the cutpoints used are standard values used in routine clinical decision making. Advanced parameters of LV function assessment (E/e $\mathrm{e}^{\prime}$ and strain) were not uniformly available and thus not incorporated into the analysis. We report all-cause mortality as the primary end point, as opposed to cardiac mortality. However, it has been demonstrated that all-cause mortality is more objective and unbiased than cardiac mortality. ${ }^{30,31}$

\section{CONCLUSIONS}

In a contemporary study of patients with AS who undergo AVR, we demonstrate that despite low 30-day mortality, approximately $20 \%$ of patients are dead at the 5-year follow-up. Along with the expected predictors such as increasing age, low GFR, and a history of cardiac surgery, different AS subtypes (and LV flow impairment) were independently associated with mortality. Patients with paradoxic severe and low LVEF severe AS had significantly worse outcomes than patients with standard severe AS. In such patients, a thorough clinical and imaging assessment needs to be performed to identify and characterize patients early, before the occurrence of advanced symptoms or LV flow impairment. Early AVR could be associated with improved outcomes in this high-risk population, although this awaits prospective determination.

\section{Conflict of Interest Statement}

Authors have nothing to disclose with regard to commercial support.

\section{References}

1. Murphy ES, Lawson RM, Starr A, Rahimtoola SH. Severe aortic stenosis in patients 60 years of age or older: left ventricular function and 10-year survival after valve replacement. Circulation. 1981;64:II184-8.

2. Smith N, McAnulty JH, Rahimtoola SH. Severe aortic stenosis with impaired left ventricular function and clinical heart failure: results of valve replacement. Circulation. 1978;58:255-64.

3. Connolly HM, Oh JK, Orszulak TA, Osborn SL, Roger VL, Hodge DO, et al. Aortic valve replacement for aortic stenosis with severe left ventricular dysfunction. Prognostic indicators. Circulation. 1997;95:2395-400.

4. Kvidal P, Bergstrom R, Horte LG, Stahle E. Observed and relative survival after aortic valve replacement. J Am Coll Cardiol. 2000;35:747-56.

5. Brogan WC III, Grayburn PA, Lange RA, Hillis LD. Prognosis after valve replacement in patients with severe aortic stenosis and a low transvalvular pressure gradient. J Am Coll Cardiol. 1993;21:1657-60.

6. Pereira JJ, Lauer MS, Bashir M, Afridi I, Blackstone EH, Stewart WJ, et al. Survival after aortic valve replacement for severe aortic stenosis with low transvalvular gradients and severe left ventricular dysfunction. J Am Coll Cardiol. 2002;39:1356-63.

7. Schwarz F, Baumann P, Manthey J, Hoffmann M, Schuler G, Mehmel HC, et al. The effect of aortic valve replacement on survival. Circulation. 1982;66: 1105-10.

8. Mihaljevic T, Nowicki ER, Rajeswaran J, Blackstone EH, Lagazzi L, Thomas J, et al. Survival after valve replacement for aortic stenosis: Implications for decision making. J Thorac Cardiovasc Surg. 2008;135:1270-9.
9. Kang DH, Park SJ, Rim JH, Yun SC, Kim DH, Song JM, et al. Early surgery versus conventional treatment in asymptomatic very severe aortic stenosis Circulation. 2010;121:1502-9.

10. Ashikhmina EA, Schaff HV, Dearani JA, Sundt TM III, Suri RM, Park SJ, et al Aortic valve replacement in the elderly: determinants of late outcome. Circulation. 2011;124:1070-8.

11. Malouf J, Le Tourneau T, Pellikka P, Sundt TM, Scott C, Schaff HV, et al. Aortic valve stenosis in community medical practice: determinants of outcome and implications for aortic valve replacement. J Thorac Cardiovasc Surg. 2012; 144:1421-7.

12. Malaisrie SC, McCarthy PM, McGee EC, Lee R, Rigolin VH, Davidson CJ, et al Contemporary perioperative results of isolated aortic valve replacement for aortic stenosis. Ann Thorac Surg. 2010;89:751-6.

13. Vahanian A, Alfieri O, Andreotti F, Antunes MJ, Baron-Esquivias G, Baumgartner H, et al. Guidelines on the management of valvular heart disease (version 2012): the joint task force on the management of valvular heart disease of the European Society of Cardiology (ESC) and the European Association for Cardio-Thoracic Surgery (EACTS). Eur J Cardiothorac Surg. 2012;42:S1-44.

14. Nishimura RA, Otto CM, Bonow RO, Carabello BA, Erwin JP III, Guyton RA et al. 2014 AHA/ACC Guideline for the Management of Patients with Valvular Heart Disease: Executive Summary: a report of the American College of Cardiology/American Heart Association Task Force on Practice Guidelines. J Am Coll Cardiol. 2014;63:2438-88.

15. Baumgartner H, Hung J, Bermejo J, Chambers JB, Evangelista A, Griffin BP et al. Echocardiographic assessment of valve stenosis: EAE/ASE recommendations for clinical practice. J Am Soc Echocardiogr. 2009;22:1-23: quiz 101-2.

16. Minners J, Allgeier M, Gohlke-Baerwolf C, Kienzle RP, Neumann FJ, Jander N. Inconsistencies of echocardiographic criteria for the grading of aortic valve stenosis. Eur Heart J. 2008;29:1043-8.

17. Pibarot P, Dumesnil JG. Low-flow, low-gradient aortic stenosis with normal and depressed left ventricular ejection fraction. J Am Coll Cardiol. 2012;60:1845-53.

18. Dumesnil JG, Pibarot P, Carabello B. Paradoxical low flow and/or low gradient severe aortic stenosis despite preserved left ventricular ejection fraction: Implications for diagnosis and treatment. Eur Heart J. 2010;31:281-9.

19. Hachicha Z, Dumesnil JG, Bogaty P, Pibarot P. Paradoxical low-flow, lowgradient severe aortic stenosis despite preserved ejection fraction is associated with higher afterload and reduced survival. Circulation. 2007;115:2856-64.

20. Otto CM. Valvular aortic stenosis: disease severity and timing of intervention. J Am Coll Cardiol. 2006;47:2141-51.

21. Ozkan A, Hachamovitch R, Kapadia SR, Tuzcu EM, Marwick TH. Impact of aortic valve replacement on outcome of symptomatic patients with severe aortic stenosis with low gradient and preserved left ventricular ejection fraction. Circulation. 2013;128:622-31.

22. Vaquette B, Corbineau H, Laurent M, Lelong B, Langanay T, de Place C, et al Valve replacement in patients with critical aortic stenosis and depressed left ventricular function: predictors of operative risk, left ventricular function recovery, and long term outcome. Heart. 2005;91:1324-9.

23. Clavel MA, Cote N, Mathieu P, Dumesnil JG, Audet A, Pepin A, et al. Paradoxical low-flow, low-gradient aortic stenosis despite preserved left ventricular ejection fraction: new insights from weights of operatively excised aortic valves. Eur Heart J. 2014;35:2655-62.

24. Clavel MA, Dumesnil JG, Capoulade R, Mathieu P, Senechal M, Pibarot P. Outcome of patients with aortic stenosis, small valve area, and low-flow, low-gradient despite preserved left ventricular ejection fraction. J Am Coll Cardiol. 2012;60:1259-67.

25. Lang RM, Bierig M, Devereux RB, Flachskampf FA, Foster E, Pellikka PA, et al Recommendations for chamber quantification: a report from the American Society of Echocardiography's Guidelines and Standards Committee and the Chamber Quantification Writing Group, developed in conjunction with the European Association of Echocardiography, a branch of the European Society of Cardiology. J Am Soc Echocardiogr. 2005;18:1440-63.

26. Nagueh SF, Appleton CP, Gillebert TC, Marino PN, Oh JK, Smiseth OA, et al Recommendations for the evaluation of left ventricular diastolic function by echocardiography. J Am Soc Echocardiogr. 2009;22:107-33.

27. Schwammenthal E, Vered Z, Agranat O, Kaplinsky E, Rabinowitz B, Feinberg MS. Impact of atrioventricular compliance on pulmonary artery pressure in mitral stenosis: an exercise echocardiographic study. Circulation. 2000;102:2378-84.

28. Zoghbi WA, Chambers JB, Dumesnil JG, Foster E, Gottdiener JS, Grayburn PA, et al. Recommendations for evaluation of prosthetic valves with 
echocardiography and Doppler ultrasound: a report from the American Society Of Echocardiography's Guidelines and Standards Committee and the Task Force On Prosthetic Valves, developed in conjunction with the American College Of Cardiology Cardiovascular Imaging Committee, Cardiac Imaging Committee Of The American Heart Association, the European Association of Echocardiography, a registered branch of the European Society Of Cardiology, the Japanese Society of Echocardiography and the Canadian Society of Echocardiography, endorsed by the American College of Cardiology Foundation, American Heart Association, European Association of Echocardiography, a registered branch of the European Society of Cardiology, the Japanese Society of Echocardiography, and Canadian Society of Echocardiography. J Am Soc Echocardiogr. 2009;22:975-1014: quiz 1082-14.
29. Rao V, Jamieson WR, Ivanov J, Armstrong S, David TE. Prosthesis-patient mismatch affects survival after aortic valve replacement. Circulation. 2000; 102:III5-9.

30. Lauer MS, Blackstone EH, Young JB, Topol EJ. Cause of death in clinical research: time for a reassessment? J Am Coll Cardiol. 1999;34:618-20.

31. Boyle CA, Decoufle P. National sources of vital status information: Extent of coverage and possible selectivity in reporting. Am J Epidemiol. 1990;131: $160-8$.

Key Words: aortic stenosis, aortic valve replacement, echocardiography, outcomes

Readers who found these articles interesting may also like to read the following papers found in recent and future issues of our sister publications, Seminars in Thoracic and Cardiovascular Surgery and Operative Techniques in Thoracic and Cardiovascular Surgery!

Acquired Cardiovascular Disease: Aortic Disease

State of the Art: Thoralf Sundt. Current Understandings and Approach to the Management of Aortic Intramural Hematomas. Semin Thorac Cardiovasc Surg. Summer 2014;26(2):123-131. 
TABLE E1. Univariable Cox proportional hazard survival analysis in the study population for the primary end point of all-cause mortality

\begin{tabular}{|c|c|c|}
\hline Variable & Hazard ratio & $P$ value \\
\hline Age (for 10-y increase) & $1.65(1.40-1.93)$ & $<.001$ \\
\hline Male gender & $1.17(0.82-1.68)$ & .35 \\
\hline Angina & $1.27(0.89-1.82)$ & .18 \\
\hline Syncope & $1.43(0.70-2.94)$ & .32 \\
\hline NYHA class & $1.84(1.51-2.25)$ & $<.001$ \\
\hline Hypertension & $1.31(0.91-1.88)$ & .13 \\
\hline Hyperlipidemia & $1.06(0.67-1.68)$ & .78 \\
\hline Diabetes mellitus & $1.16(0.81-1.67)$ & .41 \\
\hline Obstructive coronary artery disease & $1.62(1.15-2.27)$ & .006 \\
\hline Stroke & $1.42(0.71-2.95)$ & .38 \\
\hline Smoking & $1.05(0.76-1.45)$ & .76 \\
\hline Prior cardiac surgery & $2.07(1.48-2.92)$ & $<.001$ \\
\hline Atrial fibrillation & $1.51(1.05-2.16)$ & .02 \\
\hline Dialysis & $3.97(2.12-7.43)$ & $<.001$ \\
\hline Pacemaker & $1.40(1.02-1.93)$ & .04 \\
\hline Implantable defibrillator & $1.04(0.64-1.77)$ & .67 \\
\hline STS score (for every $1 \%$ increase) & $1.45(1.26-1.67)$ & $<.001$ \\
\hline Aspirin & $1.34(0.73-2.43)$ & .45 \\
\hline Beta-blockers & $0.76(0.44-1.34)$ & .34 \\
\hline ACE inhibitors & $0.79(0.57-1.08)$ & .14 \\
\hline Statins & $0.94(0.62-1.43)$ & .77 \\
\hline Diuretics & $2.64(0.85-6.55)$ & .09 \\
\hline GFR (for every 10 -unit decrease) & $1.22(1.13-1.32)$ & $<.001$ \\
\hline Indexed left atrial area & $1.03(0.86-1.43)$ & .28 \\
\hline Indexed LV end-systolic dimension & $1.37(0.98-1.95)$ & .10 \\
\hline Indexed LV mass (for $10 \mathrm{~g} / \mathrm{m}^{2}$ increase) & $1.04(1.01-1.08)$ & .03 \\
\hline LVEF & $0.97(0.96-0.98)$ & $<.001$ \\
\hline LV diastolic dysfunction & $1.63(0.80-2.70)$ & .35 \\
\hline \multicolumn{3}{|l|}{ AS subcategories } \\
\hline Standard severe & 1 & \\
\hline Paradoxic severe & $1.53(1.18-1.95)$ & $<.001$ \\
\hline Low EF severe & $2.33(1.48-3.03)$ & $<.001$ \\
\hline LV-SVI & $0.97(0.96-0.99)$ & .004 \\
\hline Aortic regurgitation & $1.09(0.95-1.26)$ & .23 \\
\hline Mitral regurgitation & $1.03(0.80-1.33)$ & .45 \\
\hline Right ventricular systolic pressure & $1.03(0.99-1.05)$ & .14 \\
\hline Indexed EOA of prosthetic valve & $0.50(0.20-0.98)$ & .03 \\
\hline Patient-prosthesis mismatch & $1.34(1.02-1.79)$ & .03 \\
\hline Time to cardiac surgery from initial visit & $1.12(1.01-1.30)$ & .04 \\
\hline Type of cardiac surgery performed & $1.12(0.95-1.39)$ & .25 \\
\hline $\begin{array}{l}\text { Type of valve prosthesis implanted } \\
\text { (bioprosthesis vs mechanical) }\end{array}$ & $1.16(0.66-2.03)$ & .58 \\
\hline
\end{tabular}

ACE, Angiotensin-converting enzyme; NYHA, New York Heart Association; STS, Society of Thoracic Surgeons; ACE, angiotensin-converting enzyme; $G F R$, glomerular filtration rate; $L V$, left ventricular; $L V E F$, left ventricular ejection fraction; $A S$, aortic stenosis; $L V-S V I$, left ventricular stroke volume index; $E O A$, effective orifice area. 